\title{
Bibliometric Analysis of South China University of Technology SCIE Articles from 2004 to 2013
}

\author{
LI Na
}

South China University of Technology Library, Guangzhou, Guangdong, China (ssjjsklina@126.com)

\begin{abstract}
Based on the SCIE (Science Citation Index Expanded) database. Using the bibliometric method to analyze the Scientific papers of South China University of Technology are indexed in SCIE database during the period of 2004-2013. And study the changing tendency of each index with time by the analysis of the main index that include the number of papers, the times cited, H-factor, the cooperant author's nationality, the source journals, the language that the articles being written and so on to reflect the overall level of scientific research in South China University of Technology during the last ten years.
\end{abstract}

Key words - SCIE, South China University Technology, scientific articles, bibliometrics

\section{4-2013 年华南理工大学 SCIE 科技论文计量分析}

\author{
李娜 \\ 华南理工大学图书馆, 广州, 广东, 中国
}

摘 要 基于 SCIE(Science Citation Index Expanded)数据库, 应用文献计量学评价方法, 对华南理工大学 2004-2013 年间被 SCIE 收录的科技论文进行分析, 通过分析被收录论文数量、 $\mathrm{H}$ (影响) 因子、被引频次、合著国家或地区、来源期刊等指标, 研究各 指标随着时间的变化趋势, 客观反映出华南理工大学近十年科研水平的总体状况。

关键词 SCIE, 华南理工大学, 科技论文, 文献计量学

科学引文索引(Science Citation Index Expanded, 简称 SCI-EXPANDED) 是由美国科技情报学专家尤金·加菲尔德 博士(Eugene Garfield)所创立的, 是美国科学信息研究所 (Institute For Scientific Information, 简称 ISI)的主要引文索 引数据库 ${ }^{[1]}$ 。SCIE 数据库覆盖全世界最重要和最有影响力 的研究成果, 是国际通行的一种对自然科学基础研究成果 进行评价的比较客观、定量和易操作指标, 它在衡量国家、 科研机构或大学的科研实力、评价科研人员的学术水平等 方面发挥着一定作用 ${ }^{[2]}$ 。科技论文的数量和质量是科技产 出的重要指标之一,一所高校被 SCIE 数据库收录科技论文 的多少, 被视为其科研水平、科技实力和综合研究能力的 重要指标 ${ }^{[3]}$ 。另一方面, 由于 SCIE 能够及时、准确地反映 基础科学的重大突破、学科前沿的最新研究动态和热点、 难点问题, 在学术界占有重要地位。因此, 被 SCIE 收录 的科技论文数量和质量已经成为国际公认的评价科研实力 与水平, 反映学术地位的重要指标 ${ }^{[4]}$ 。

鉴于 SCIE 收录论文的权威性和科学性, 本文对华南理 工大学 2004-2013 年 SCIE 科技论文状况进行计量分析, 主

基金项目：中央高校基本科研业务费资助项目（2014GM07）
要是将华南理工大学近十年 SCIE 科技论文进行纵向自 身对比, 分析华南理工大学 SCIE 科技论文的发展情况, 总结其内在规律和发展趋势, 从而为科技管理部门科学 决策及加强 SCIE 收录论文的管理提供有益借鉴, 同时 正确引导 SCIE 收录论文的评价作用, 提高科技决策的 科学性, 从而切实提高华南理工大学科研论文的数量和 质量。

\section{1. 数据来源}

本文基于 Web of Science 检索平台中的 SCIE(Science Citation Index Expanded)数据库, 研究对 象是 2004-2013 年华南理工大学学者发表在 SCIE 收录 期刊上的科技论文。在 SCIE 数据库中, 以作者单位(华 南理工大学）和年份进行交叉检索。例如：用“s china univ technol”作为“地址”的检索词, 分别以“2004”、 “2005”、“2006”、“2007”、“2008”、“2009”、“2010”、 “2011”、“2012”、“2013”作为“出版年”的检索词, 即对 华南理工大学 2004-2013 年期间发表的科技论文逐年 进行计量分析, 检索时间为 2014 年 1 月 3 日。然后利 用 web of science 数据库分析功能对检索到的文献进行 
计量分析。这里需要说明的是: 其一, 本次检索未包括华 南理工大学科研人员署其他单位名称发表的论文; 其二, 由于各期刊发行时间与数据库更新时间不同步, 2013 年华 南理工大学 SCI E 收录的论文可能会有个别遗漏; 其三, 论文作者未区分是否为第一作者。

\section{2. 数据统计}

在科学研究中, 不同的国家、地区以及单位之间的合 作与交流越来越重要, 本文将华南理工大学 SCIE 科技论文 按合作者的国别和机构进行了分类: 独著 SCIE 科技论文、

表 1 华南理工大学 SCIE 科技论文总体情况统计表

\begin{tabular}{|c|c|c|c|c|c|}
\hline 年份 & $\begin{array}{c}\text { 论文 } \\
\text { 篇数 }\end{array}$ & $\mathrm{H}$ 因子 & $\begin{array}{c}\text { 总被引 } \\
\text { 频次 }\end{array}$ & $\begin{array}{c}\text { 平均被引 } \\
\text { 频次 }\end{array}$ & $\begin{array}{c}\text { 国际 } \\
\text { 引用率 }\end{array}$ \\
\hline 2004 & 350 & 38 & 5726 & 16.41 & $46.04 \%$ \\
\hline 2005 & 523 & 38 & 6182 & 11.87 & $46.14 \%$ \\
\hline 2006 & 805 & 43 & 9076 & 11.34 & $45.49 \%$ \\
\hline 2007 & 924 & 46 & 10912 & 11.90 & $45.42 \%$ \\
\hline 2008 & 1084 & 42 & 11585 & 10.75 & $47.47 \%$ \\
\hline 2009 & 1364 & 45 & 14549 & 10.78 & $46.19 \%$ \\
\hline 2010 & 1415 & 43 & 14361 & 10.26 & $50.85 \%$ \\
\hline 2011 & 1776 & 32 & 10042 & 5.78 & $43.76 \%$ \\
\hline 2013 & 1948 & 20 & 5551 & 3.0 & $44.13 \%$ \\
\hline
\end{tabular}

表 2 华南理工大学独著 SCIE 科技论文统计表

\begin{tabular}{|c|c|c|c|c|c|}
\hline 年份 & $\begin{array}{c}\text { 论文 } \\
\text { 篇数 }\end{array}$ & H 因子 & $\begin{array}{c}\text { 总被引 } \\
\text { 频次 }\end{array}$ & $\begin{array}{c}\text { 平均被引 } \\
\text { 频次 }\end{array}$ & $\begin{array}{c}\text { 国际 } \\
\text { 引用率 }\end{array}$ \\
\hline 2004 & 119 & 17 & 1374 & 11.59 & $45.70 \%$ \\
\hline 2005 & 198 & 26 & 2442 & 12.38 & $48.34 \%$ \\
\hline 2006 & 335 & 29 & 3349 & 10.05 & $43.48 \%$ \\
\hline 2007 & 405 & 30 & 4292 & 10.69 & $43.66 \%$ \\
\hline 2008 & 468 & 33 & 5228 & 11.23 & $47.08 \%$ \\
\hline 2009 & 556 & 31 & 5534 & 10.04 & $43.16 \%$ \\
\hline 2010 & 590 & 29 & 4933 & 8.47 & $41.17 \%$ \\
\hline 2011 & 670 & 20 & 3117 & 4.74 & $38.36 \%$ \\
\hline 2012 & 780 & 16 & 2326 & 3.12 & $42.81 \%$ \\
\hline 2013 & 707 & 6 & 210 & 0.42 & $42.78 \%$ \\
\hline
\end{tabular}

分别从表 1、表 2、表 3、表 4 中可以看出, 在上述 十个统计年份中, 以华南理工大学为作者单位发表的全 部 SCIE 科技论文、独著 SCIE 科技论文、国内合著 SCIE 科技论文以及国际合著 SCI 科技论文的数量、 $\mathrm{H}$ 因子、 总被引频次、平均被引频次、国际引用率等指标均有不 同程度的变化。
国内合著 SCIE 科技论文、国际合著 SCIE 科技论文。 独著 SCIE 科技论文是指所有作者都为华南理工大学学 者的 SCIE 科技论文, 国内合著 SCIE 科技论文是指华 南理工大学学者与国内其它单位学者合著的 SCIE 科技 论文, 国际合著 SCIE 科技论文是华南理工大学学者与 国外学者合著的 SCIE 科技论文。现将 2004-2013 年华 南理工大学 SCIE 科技论文总体情况、独著情况、国内 合著情况、国际合著情况分别进行统计, 详见表 1、表 2、表 3 和表 4 。

表 3 华南理工大学国内合著 SCIE 科技论文统计表

\begin{tabular}{|c|c|c|c|c|c|}
\hline 年份 & $\begin{array}{c}\text { 论文 } \\
\text { 篇数 }\end{array}$ & $\mathrm{H}$ 因子 & $\begin{array}{c}\text { 总被引 } \\
\text { 频次 }\end{array}$ & $\begin{array}{c}\text { 平均被引 } \\
\text { 频次 }\end{array}$ & $\begin{array}{c}\text { 国际 } \\
\text { 引用率 }\end{array}$ \\
\hline 2004 & 162 & 24 & 2337 & 14.46 & $40.71 \%$ \\
\hline 2005 & 258 & 25 & 2423 & 9.43 & $39.61 \%$ \\
\hline 2006 & 354 & 27 & 3064 & 8.68 & $39.25 \%$ \\
\hline 2008 & 401 & 34 & 4664 & 11.67 & $42.66 \%$ \\
\hline 2009 & 518 & 28 & 4341 & 8.42 & $42.19 \%$ \\
\hline 2010 & 518 & 24 & 4123 & 8.00 & $42.67 \%$ \\
\hline 2011 & 711 & 20 & 4202 & 5.54 & $39.94 \%$ \\
\hline 2012 & 824 & 14 & 1940 & 2.43 & $37.16 \%$ \\
\hline 2013 & 952 & 6 & 348 & 0.45 & $30.93 \%$ \\
\hline
\end{tabular}

表 4 华南理工大学国际合著 SCIE 科技论文统计表

\begin{tabular}{|c|c|c|c|c|c|}
\hline 年份 & $\begin{array}{c}\text { 论文 } \\
\text { 篇数 }\end{array}$ & $\mathrm{H}$ 因子 & $\begin{array}{c}\text { 总被引 } \\
\text { 频次 }\end{array}$ & $\begin{array}{c}\text { 平均被引 } \\
\text { 频次 }\end{array}$ & $\begin{array}{c}\text { 国际 } \\
\text { 引用率 }\end{array}$ \\
\hline 2004 & 69 & 22 & 2021 & 29.33 & $51.30 \%$ \\
\hline 2005 & 67 & 19 & 1322 & 19.75 & $52.33 \%$ \\
\hline 2006 & 116 & 27 & 2681 & 23.16 & $52.92 \%$ \\
\hline 2007 & 118 & 25 & 1981 & 16.84 & $51.27 \%$ \\
\hline 2008 & 217 & 29 & 2930 & 13.57 & $47.96 \%$ \\
\hline 2009 & 290 & 31 & 4727 & 16.41 & $51.22 \%$ \\
\hline 2010 & 307 & 29 & 5340 & 17.50 & $62.87 \%$ \\
\hline 2011 & 335 & 24 & 2783 & 8.42 & $50.62 \%$ \\
\hline 2012 & 344 & 15 & 1368 & 4.06 & $50.44 \%$ \\
\hline 2013 & 424 & 7 & 306 & 0.85 & $53.08 \%$ \\
\hline
\end{tabular}

\section{3. 数据分析}

\section{1 论文数量}

2004-2013 年, 以华南理工大学为作者单位, 共有 12272 篇论文被 SCIE 收录。其中, 独著的科技论文共 4828 篇, 国内合著的科技论文共 5097 篇, 国际合著的科技论 文共 2287 篇。 


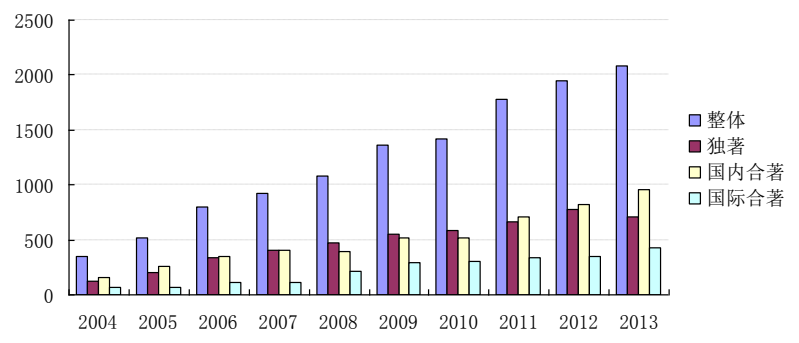

图 1 2004-2013 年华南理工大学 SCIE 科技论文数量分布

从图 1 可以看出, 华南理工大学 SCIE 科技论文数量 呈现逐年增长的趋势, 就整体情况而言, 2008 年 SCIE 科技论文数量突破 1000 篇, 2013 年 SCIE 科技论文数量 突破 2000 篇, 这说明从 2008 到 2013 这五年期间华南理 工大学 SCIE 科技论文数量在迅猛的增长, 这样快速的增 长幅度也使得华南理工大学 SCIE 科技论文总量在全国 排名不断攀升。独著 SCIE 科技论文、国内合著 SCIE 科 技论文、国际合著 SCIE 科技论文三者进行比较, 独著 SCIE 科技论文数量和国内合著 SCIE 科技论文数量相 当, 但国际合著 SCIE 科技论文数量远远小于独著 SCIE 科技论文数量和国内合著 SCIE 科技论文数量, 由此可见 华南理工大学学者发表的 SCIE 科技论文还是以独著和 国内合著为主, 在国际合作与交流方面还有待加强。

基于表 1 、表 $2 、$ 表 3 、表 4 的统计数据, 进一步对 总体、独著、国内合著以及国际合著 SCIE 科技论文数量 增长率进行比较分析 (科技论文增长率 $=($ 目标年份指标 一上一年份指标) /上一年份指标), 详见图 2 。

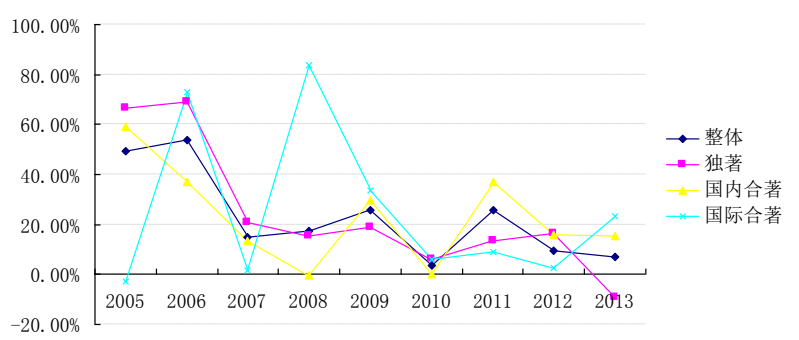

图 2 2004-2013 年华南理工大学 SCIE 科技论文增长率变化情况

从图 2 中可以看出, 独著 SCIE 科技论文数量变化规 律、国内合著 SCIE 科技论文变化规律与整体 SCIE 科技 论文变化规律基本保持一致。华南理工大学独著 SCIE 科技论文占总量的 $39.34 \%$, 国内合著的 SCEI 科技论文 占总量的 $41.53 \%$, 二者之和占论文总量的 $80.87 \%$, 由此 可见华南理工大学 SCIE 科技论文的增长主要来源于独 著和国内合著 SCIE 科技论文的增长。

国际合著 SCIE 科技论文数量变化规律相对而言呈 现出明显的波动性, 2006 年增长率为 73.13\%, 2008 年 增长率为 $83.90 \%, 2009$ 的增长率 $33.64 \% ， 2013$ 年增长 率为 $23.26 \% ， 2005 、 2007 、 2010 、 2011 、 2012$ 这五个年
份增长率基本保持在[-3\%, 10\%]区间内变化。

\section{$3.2 \mathrm{H}$ (影响)因子}

$\mathrm{H}$ 因子(又称 $\mathrm{H}$ 指数) 是美国物理学家 Hirsch 于 2005 年提出用于“评价科学家的科研绩效”。 $\mathrm{H}$ 因子的物理含 义可简单的表述为: “有 $\mathrm{h}$ 篇论文被引用了不少于 $\mathrm{h}$ 次”。 $\mathrm{H}$ 因子主要是一个影响力判定参数, 其特点是关注科学 家发表了多少有影响力的论文 ${ }^{[5]}$ 。

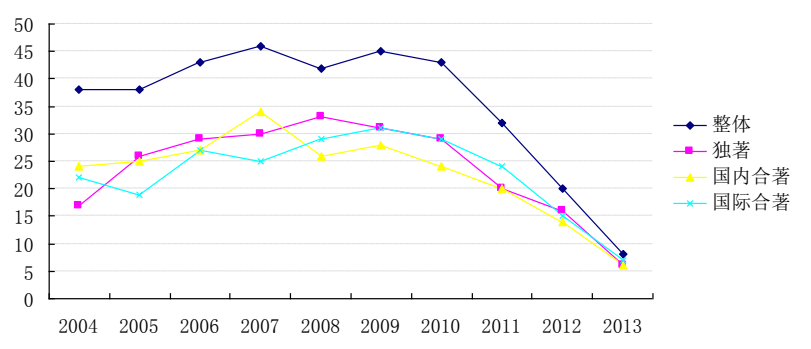

图 3 2004-2013 年华南理工大学 SCIE 科技论文 H 因子分布情况

从图 3 中可以看出, 从 2004 到 2009 年 SCIE 科技论 文 H 因子呈现稳步上涨趋势, 从 2009 到 2013 年 SCIE 科技论文 H 因子呈现下降趋势, 尤其 2011、2012、2013 这三个统计年份, SCIE 科技论文的 H 因子急剧下降, 因 为这三个年份距离数据统计时间最短。 $\mathrm{H}$ 因子的大小与 科技论文被频次成正比关系, 而被引频次的增加需要时 间的积累, 科技论文发表的时间越短, 将导致科技论文 被引频次越低, 所以 $\mathrm{H}$ 因子也越低。

从图 3 中还可以看出, 从 2004-2007 这四个统计年 份, 国内合著 SCIE 科技论文 $\mathrm{H}$ 因子大于国际合著 SCIE 科技论文 H 因子; 从 2008-2013 这六个统计年份, 国内 合著 SCIE 科技论文 H 因子小于国际合著 SCIE 科技论 文 $\mathrm{H}$ 因子。这说明近年来华南理工大学国际合著 SCIE 科技论文的质量远远高于国内合著 SCIE 科技论文的质 量, 由此可见华南理工大学在国际学术界的影响力不断 地增强。

\section{3 被引频次}

科技论文的被引情况是衡量其质量和国际影响力的 重要标准。对某一研究领域来讲, 质量较高的论文一般 都具有较高被引频次 ${ }^{[4]}$ 。在对被引频次进行分析时, 为 了更加客观准确地反映科技论文质量, 将自引排除在研 究范围之外。基于表 1、表 2、表 3、表 4 的统计数据, 分别对 2004-2013 年华南理工大学总体、独著、国内合 著、国际合著 SCIE 科技论文的总被引频次、平均被引频 次和国际引用率进行分析。 


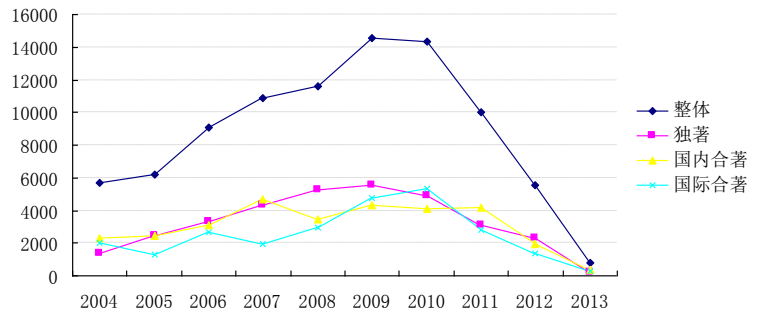

图 4 2004-2013 年华南理工大学 SCIE 科技论文总被引频次分布 情况

将图 4 与图 3 进行比较可以看出, 科技论文总被引 频次变化趋势与科技论文 $\mathrm{H}$ 因子变化趋势基本相同, 由 此也验证了 $\mathrm{H}$ 因子与科技论文被引频次是成正比关系 的。

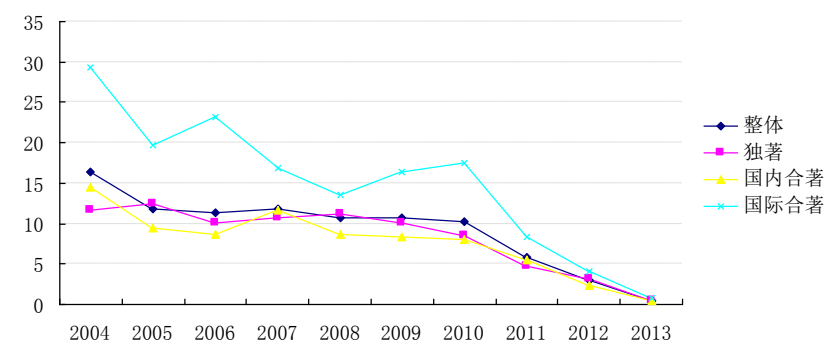

图 5 2004-2013 年华南理工大学 SCIE 科技论文平均被引频次分 布情况

从图 5 中可以看出, 华南理工大学国际合著 SCIE 科技论文平均被引频次明显高于国内合著 SCIE 科技论 文以及独著 SCIE 科技论文, 但是差距随着时间的推移在 不断变小。

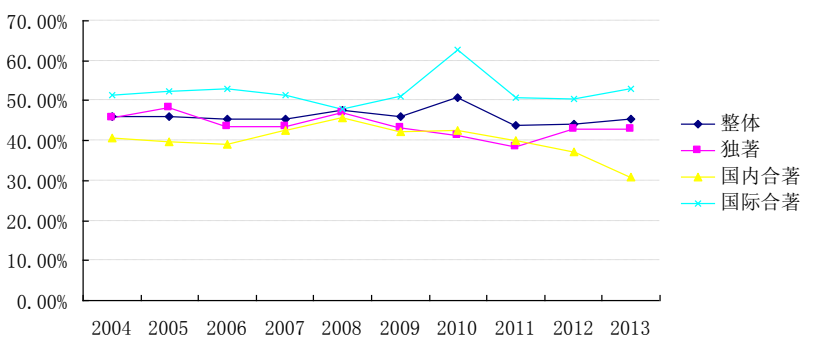

图 6 2004-2013 年华南理工大学 SCIE 科技论文国际引用率变化 情况

图 6 反映了华南理工大学 SCIE科技论文国外被引比 例的变化情况, 科技论文被国外的学者所引用, 说明该 论文对国外的科学研究起到了参考、借鉴和学习的作用, 表明其在国外产生了一定的影响力。从图 6 中可以看出, 华南理工大学国际合著 SCIE 科技论文的国际影响力大 于国内合著 SCIE 科技论文以及独著 SCIE 科技论文。另
外,华南理工大学总体 SCIE 科技论文国外被引比例始终 保持在一个平稳的态势。

\section{4 合著国家或地区}

表 4 已对华南理工大学国际合著 SCIE 科技论文数量 进行了统计, SCIE 科技论文数量呈现了增长趋势。现选 取与南理工大学合著 SCIE 科技论文且排名前十位的国 家或地区进行统计分析。

表 5 2004-2013 年华南理工大学 SCIE 科技论文前 10 位合著国 家或地区统计表

\begin{tabular}{|c|c|c|c|c|c|}
\hline 排名 & 国家或地区 & 论文篇数 & $\begin{array}{c}\text { 总被引 } \\
\text { 频次 }\end{array}$ & $\begin{array}{c}\text { 平均被引 } \\
\text { 频次 }\end{array}$ & $\mathrm{P}$ \\
\hline 1 & 美国 & 886 & 8844 & 10.65 & $38.538 \%$ \\
\hline 2 & 澳大利亚 & 217 & 2478 & 12.01 & $9.439 \%$ \\
\hline 3 & 日本 & 217 & 2042 & 9.98 & $9.439 \%$ \\
\hline 4 & 加拿大 & 205 & 2282 & 11.45 & $8.917 \%$ \\
\hline 5 & 新加坡 & 197 & 2777 & 14.64 & $8.569 \%$ \\
\hline 6 & 德国 & 149 & 2866 & 20.38 & $6.481 \%$ \\
\hline 7 & 英国 & 138 & 2856 & 20.98 & $6.003 \%$ \\
\hline 8 & 韩国 & 67 & 732 & 11.09 & $2.914 \%$ \\
\hline 9 & 法国 & 66 & 2113 & 32.45 & $2.871 \%$ \\
\hline 10 & 台湾 & 61 & 305 & 5.61 & $2.653 \%$ \\
\hline
\end{tabular}

注释: P=Percentage 是将合著国家的合著 SCIE 科技论文数量除以整体 合著 SCIE 科技论文数量, 得到各国合著 SCIE 科技论文占总合著 SCIE 科技论文的百分比。

2004-2013 年, 华南理工大学与 58 个国家和地区分 别进行了科研合作。从表 5 可以看出，合作最多的国家 是美国，占总数的 $38.538 \%$; 合作较多的国家是澳大利 亚、日本、加拿大、新加坡、德国和英国, 分别占总数 的 $9.439 \% 、 9.439 \% 、 8.917 \% 、 8.569 \% 、 6.481 \%$ 和 $6.003 \%$; 其次进行合作的国家和地区还有韩国、法国、 台湾等。由此可见, 华南理工大学与科技强国之间的合 作与交流相对密切。

在合著 SCIE 科技论文质量方面, 与排名前十个国 家合著 SCIE 科技论文, 除台湾以外, 平均被引次数均超 过 10 次, 其中最高的为法国 (32.45 次), 最低的为美国 (10.65 次)。由此可见, 与华南理工大学合作情况密切 的国家 SCIE 科技论文质量也明显高于其它国家合著的 SICE 科技论文。

\section{5 来源期刊}

2004-2013 年, 华南理工大学被 SCIE 收录的科技论 文分布在 600 多种期刊上, 现选取排名前 10 位的期刊进 行列表分析。 
表 6 2004-2013 年华南理工大学 SCIE 科技论文前 10 位来源期刊统计表

\begin{tabular}{|l|l|l|l|l|l|}
\hline 排名 & 来源期刊 & 论文篇数 & 影响因子 & 占总收录比例(\%) & 国别 \\
\hline 1 & JOURNAL OF APPLIED POLYMER SCIENCE & 222 & 1.395 & 1.802 & 美国 \\
\hline 2 & ACTA PHYSICA SINICA & 138 & 1.016 & 1.120 & 中国 \\
\hline 3 & BIORESOURCES & 130 & 1.309 & 1.055 & 美国 \\
\hline 4 & BIORESOURCE TECHNOLOGY & 120 & 4.750 & 0.974 & 荷兰 \\
\hline 5 & $\begin{array}{l}\text { TRANSACTIONS OF NONFERROUS METALS SOCIETY OF } \\
\text { CHINA }\end{array}$ & 107 & 0.917 & 0.868 & 中国 \\
\hline 6 & CARBOHYDRATE POLYMERS & 104 & 3.479 & 0.844 & 英国 \\
\hline 7 & JOURNAL OF ALLOYS AND COMPOUNDS & 103 & 2.390 & 0.836 & 瑞士 \\
\hline 8 & JOURNAL OF AGRICULTURAL AND FOOD CHEMISTRY & 101 & 2.906 & 0.820 & 美国 \\
\hline 9 & RARE METAL MATERIALS AND ENGINEERING & 97 & 0.160 & 0.787 & 中国 \\
\hline 10 & APPLIED SURFACE SCIENCE & 91 & 2.112 & 0.738 & 荷兰 \\
\hline
\end{tabular}

按收录华南理工大学 SCIE 科技论文数量对来源期 刊进行排序, 表 6 列出来了排名前 10 位的期刊名称及其 相关信息, 这些期刊多数是外国期刊, 其中中国出版的 期刊有 3 个。排名第一的期刊为 JOURNAL OF APPLIED POLYMER SCIENCE, 其十年间收录华南理工大学 SCIE 科技论文总数为 222 篇，约占总收录的 $1.802 \%$ ，这一期 刊主要涉及工程与材料、有机高分子材料、化学科学、 高分子科学等, 由此表明华南理工大学在高分子材料科 学研究方面具有很大的潜力。

期刊影响因子(Index Factor, IF) 是一定时间内期刊论 文的平均被引用率。IF 的大小可以反映学术期刊的有效 度、利用度、学术性和质量。刊物所载论文量、时间和 论文被引次数是构成 IF 的基本要素 ${ }^{[6]}$ 。期刊影响因子是 表示期刊学术水平的重要指标。从表 6 中可以看出, 排 名前十的来源期刊中，影响因子最高的期刊为 BIORESOURCE TECHNOLOGY, 影响因子是 4.750, 其 十年间收录华南理工大学科技论文总数为 120 篇, 约占 总收录的 $0.868 \%$, 位于期刊排名第 4 位, 这一期刊涉及 生物能源、生物资源利用、生物资源制备技术开发、青 霉菌等, 由此表明华南理工大学在生物能源科学研究方 面具有很高的影响力。

\section{4. 结论与建议}

2004-2013 年, 华南理工大学 SCIE 科技论文数量指 标呈大幅增长趋势, 国际合著 SCIE 科技论文数量分别小 于独著 SCIE 科技论文数量和国内合著 SCIE 科技论文数 量, 但近年来华南理工大学国际合著 SCIE 科技论文的质 量远远高于国内合著 SCIE 科技论文的质量, 由此可见华 南理工大学在国际学术界的影响力在不断地增强。基于 上述分析及其研究特点, 提出以下几点建议:

(1) 华南理工大学在科技发展战略上应鼓励国际学 术合作, 尤其努力促进与科技强国之间的学术合作, 这
样才能提高华南理工大学的科研质量, 同时提升华南理 工大学国际学术影响力。

(2) 在进行人才科技评价时，除了参考 SCIE 科技论 文数量指标以外, 更应该注重质量指标的评价, 如 SCIE 科技论文被引频次、SCIE 科技论文 $\mathrm{H}$ 因子、SCIE 科技 论文来源期刊影响因子等其它指标, 促进华南理工大学 SCIE 科技论文质量的增长。

(3) 积极鼓励学者加强外语学习, 提高华南理工大学 学者整体外语水平。通过外语读、写能力的提高, 有利 于及时跟踪国际学术前沿的科技动态, 加强华南理工大 学英文科技论文的总体数量的提升。

\section{参考文献 (References)}

[1] Garfield E.The Citation Index as a subject index. Current Contents, 1974, 18:527.

[2] ZHANG Yu. SCI Statistic and Analysis of Articles about Liaoning University from 1958 to 2003. JOURNAL OF LIAO NING UNIVERSITY: Natural Sciences Edition, 2005, 32(1): 82-85.

[3] QIU Jia-yi. Analysis on the Situation of Zhongshan University's Papers Collected and Cited by SCI (Science Citation Index) in 2001-2006. Sci-Tech Information Development \& Economy, 2008, 18(3): 3-5.

[4] DONG Zheng-e, XU Hui-hua, CHEN Hui-lan. Development of Scientific Research in Donghua University Based on the SCI-E Database. Journal of Donghua University (Natural Science), 2009, 35(5):601-608.

[5] H-factor.[2014-01-20].http://baike.baidu.com/view/1719083.h tm? fr=aladdin\#4.

[6] Egghe L, Rousseau R. The influence of publication delays on the observed aging distribution of scientific literature. JOURNAL OF THE AMERICAN SOCIETY FOR INFORMATION SCIENCE, 2000, 51(2): 158-165. 